\title{
COMPUTATION OF CHARGED-PARTICLE TRANSFER MAPS FOR GENERAL FIELDS AND GEOMETRIES USING ELECTROMAGNETIC BOUNDARY-VALUE DATA
}

\author{
A.J. Dragt and T.J. Stasevich, University of Maryland, College Park, Maryland 20742, USA* \\ P. Walstrom, Los Alamos National Laboratory, Los Alamos, New Mexico, 87545 USA
}

\section{Abstract}

The passage of a charged particle through a region of nonvanishing electromagnetic fields (e.g., a bending magnet, multipole magnet, spectrometer, electrostatic lens, electromagnetic velocity separator, etc.) can be described by a transfer map [1]. In the magnetic case, computation of this map in canonical coordinates requires a knowledge of the vector potential and its multiple derivatives within the region. This information is shown to be calculable from boundary data with the aid of Helmholtz's theorem and a novel application of the Dirac monopole vector potential. This can be done without solving Laplace's equation within the region, a generally formidable problem. Using the methods to be described, modules could be added to existing electromagnetic codes that would produce reliably, when requested, associated transfer maps to any desired order for arbitrary static charged-particle beamline elements.

\section{INTRODUCTION}

Look at the volume $V^{\prime}$ shown below. It consists of a bent rectangular box flanked by straight arms. As illustrated, this volume is suitable for enclosing a reference trajectory through a bending magnet. The bent box encloses the bent part of the trajectory produced by the main bending field, and the straight arms enclose the entering and exiting parts of the trajectory in the fringe-field regions. Suppose all 3 components of the magnetic field $\boldsymbol{B}$ are known on the surface (boundary) $S^{\prime}$ of the volume (either as a result of direct measurement or from running some 3-dimensional electromagnetic code). This paper describes how the transfer map $\mathcal{M}$ for trajectories through such an element can be calculated from this boundary data.

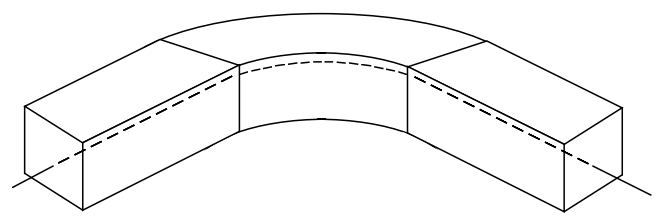

Figure 1: A volume $V^{\prime}$ surrounding a reference trajectory for a bending magnet.

* Work supported in part by DOE Grant DEFG0296ER40949.

\section{MOTION IN A STATIC MAGNETIC FIELD}

The methods of this paper can be applied to both static electric and static magnetic fields, and combinations of the two. For purposes of exposition, we will consider the case of magnetic fields.

In Cartesian coordinates and with the time $t$ as the independent variable, the Hamiltonian $H$ for motion of a particle of charge $q$ in a magnetic field is given by the relation

$$
H=\left[m^{2} c^{4}+c^{2}(\boldsymbol{p}-q \boldsymbol{A})^{2}\right]^{1 / 2} .
$$

Here $\boldsymbol{A}$ is the vector potential associated with the $\boldsymbol{B}$ field by the relation $\boldsymbol{B}=\nabla \times \boldsymbol{A}$.

For the purposes of generating maps it is more convenient to use one of the coordinates, say the $z$ coordinate, as the independent variable and to treat the time $t$ and its canonically conjugate momentum $p_{t}$ as dependent variables. With this choice of phase-space coordinates, the Hamiltonian $K$ for motion in a magnetic field is given by the relation [1]

$K=-\left[p_{t}^{2} / c^{2}-m^{2} c^{2}-\left(p_{x}-q A_{x}\right)^{2}-\left(p_{y}-q A_{y}\right)^{2}\right]^{1 / 2}-q A_{z}$.

Replace the symbol $z$ by $\tau$ in (2), and let the symbol $z$ now denote the collection of phase-space variables

$$
z=\left(x, p_{x}, y, p_{y}, t, p_{t}\right)
$$

Let $z^{d}$ be the design (reference) trajectory and introduce deviation variables $\zeta$ by the rule

$$
z=z^{d}+\zeta
$$

Suppose $K\left[z^{d}(\tau)+\zeta, \tau\right]$ is expressed as a power series in $\zeta$ by writing the expansion

$$
K\left[z^{d}(\tau)+\zeta, \tau\right]=\sum_{m=0}^{\infty} K_{m}(\zeta, \tau)
$$

where each quantity $K_{m}(\zeta, \tau)$ is a homogeneous polynomial of degree $m$ in the components of $\zeta$. It can be shown that the design orbit is determined by $K_{1}$, the linear part of the transfer map about the design orbit is determined by $K_{2}$, and the higher-order (nonlinear) parts of the transfer map are determined by the $K_{m}$ with $m \geq 3$ [1]. Evidently, determination of the $K_{m}$ requires a knowledge of $\boldsymbol{A}$ and its multiple derivatives. 


\section{CALCULATING THE VECTOR POTENTIAL FROM BOUNDARY DATA}

Assume that $\boldsymbol{B}$ is divergence and curl free within $V^{\prime}$. With the aid of Helmholtz's theorem, the use of Dirac's magnetic monopole vector potential, and various vector identities, it can be shown that $\boldsymbol{A}$ has the representation

$$
\boldsymbol{A}=\boldsymbol{A}^{n}+\boldsymbol{A}^{t}
$$

where

$$
\begin{gathered}
\boldsymbol{A}^{n}(\boldsymbol{r})=(1 / 4 \pi) \int_{S^{\prime}}\left[\boldsymbol{n}\left(\boldsymbol{r}^{\prime}\right) \cdot \boldsymbol{B}\left(\boldsymbol{r}^{\prime}\right)\right] \boldsymbol{G}^{n}\left[\boldsymbol{r} ; \boldsymbol{r}^{\prime}, \boldsymbol{n}\left(\boldsymbol{r}^{\prime}\right)\right] d S^{\prime} \\
\boldsymbol{A}^{t}(\boldsymbol{r})=(1 / 4 \pi) \int_{S^{\prime}} \Psi\left(\boldsymbol{r}^{\prime}\right) \boldsymbol{G}^{t}\left[\boldsymbol{r} ; \boldsymbol{r}^{\prime}, \boldsymbol{n}\left(\boldsymbol{r}^{\prime}\right)\right] d S^{\prime}
\end{gathered}
$$

Here $G^{n}$ and $G^{t}$ are the kernels

$$
\begin{gathered}
\boldsymbol{G}^{n}\left(\boldsymbol{r} ; \boldsymbol{r}^{\prime}, \boldsymbol{m}\right)=\frac{\boldsymbol{m} \times\left(\boldsymbol{r}-\boldsymbol{r}^{\prime}\right)}{\left[\left|\boldsymbol{r}-\boldsymbol{r}^{\prime}\right|-\boldsymbol{m} \cdot\left(\boldsymbol{r}-\boldsymbol{r}^{\prime}\right)\right]\left|\boldsymbol{r}-\boldsymbol{r}^{\prime}\right|}, \\
\boldsymbol{G}^{t}\left[\boldsymbol{r} ; \boldsymbol{r}^{\prime}, \boldsymbol{n}\left(\boldsymbol{r}^{\prime}\right)\right]=\boldsymbol{n}\left(\boldsymbol{r}^{\prime}\right) \times \nabla^{\prime} \frac{1}{\left|\boldsymbol{r}-\boldsymbol{r}^{\prime}\right|} .
\end{gathered}
$$

The vector $\boldsymbol{n}$ is the outward normal to $S^{\prime}$, and $\Psi$ is the scalar potential such that $\boldsymbol{B}=\nabla \Psi$ in $V^{\prime}[2,3,4]$. Note that $\boldsymbol{A}^{n}$ depends only on the normal component of $\boldsymbol{B}$ on $S^{\prime}$, and $\boldsymbol{A}^{t}$ depends only on the tangential component of $\boldsymbol{B}$ on $S^{\prime}$. (The value of $\Psi$ on $S^{\prime}$ can be determined by line integrals of the tangential component of $\boldsymbol{B}$ on $S^{\prime}$.) Note also that $\boldsymbol{A}$ has been calculated from boundary data without having to solve Laplace's equation in the domain $V^{\prime}$.

\section{IMPLEMENTATION AND EXPECTATIONS}

Evidently the kernels $G^{n}$ and $G^{t}$ are analytic in the components of $\boldsymbol{r}$ for $\boldsymbol{r} \in V^{\prime}$ and $\boldsymbol{r}^{\prime} \in S^{\prime}$. Therefore $\boldsymbol{G}^{n}$ and $G^{t}$ can be expanded in convergent Taylor series using Truncated Power Series Algebra (TPSA) methods [1], and the integrals (7) and (8) can be evaluated numerically to yield corresponding convergent Taylor Series for $\boldsymbol{A}^{n}$ and $\boldsymbol{A}^{t}$. These Taylor series may then be employed in (5) to yield, again using TPSA, the polynomials $K_{m}$. Finally, by already established methods, these polynomials can be used to determine the design orbit $z^{d}$ and the transfer map $\mathcal{M}$ about this orbit $[5,1]$.

It can be shown, as a result of the properties of $G^{n}$ and $\boldsymbol{G}^{t}$, that the representation (6) satisfies the desirable relations $\nabla \cdot \boldsymbol{A}=0$ and $\nabla \times \boldsymbol{B}=\nabla \times \nabla \times \boldsymbol{A}=0$ no matter what the values of $\boldsymbol{B}$ and $\Psi$ are on $S^{\prime}$, and no matter how poorly the integrals are evaluated (say numerically). In addition, the kernels $G^{n}$ and $G^{t}$ are smoothing. Consequently, we expect that the accuracy of the resulting design orbit and associated transfer map will be relatively robust against errors in the surface data $[6,7]$.

Developmental numerical code has been written to test the methods just described, and it has been verified that the method works to high accuracy for various special cases for which the design orbit and transfer map can be computed by other means. Work is currently in progress to examine in detail to what extent the method is indeed robust against errors in the surface data, and to write efficient production code including code for parallel processors.

\section{CONCLUSIONS}

A new method has been developed for the computation of charged-particle transfer maps for general fields and geometries based on the use of surface (boundary-value) data. The method requires a knowledge of all 3 field components on the surface (or, equivalently, the value of the normal field component and the scalar potential on the surface). These surface values are convolved with explicitly known and geometry-independent kernels to produce interior fields. The kernels themselves are obtained by the use of Helmholtz's theorem and Dirac magnetic monopole vector potentials. The resulting interior fields satisfy the Maxwell equations exactly and are analytic functions of position even if the surface data contains errors and/or the convolutions are only performed approximately. The resulting transfer maps are expected to be optimally robust against computational and/or measurement errors. Using these methods, modules can be added to existing numerical electromagnetic field-solving codes that would produce reliably, when requested, design orbits and associated transfer maps to any desired order for arbitrary static chargedparticle beamline elements.

\section{REFERENCES}

[1] A. J. Dragt, Lie Methods for Nonlinear Dynamics with Applications to Accelerator Physics, University of Maryland Physics Department technical report, (2001).

[2] R. Plonsey and R.E. Collin, Principles and Applications of Electromagnetic Fields, The Maple Press Company, PA, 1961.

[3] J. D. Jackson, Classical Electrodynamics, 3rd Ed., John Wiley and Sons, N.Y., 1999, p. 278.

[4] J. Schwinger, L. DeRaad, et al., Classical Electrodynamics, Perseus Books, 1998, Chapters 2 and 30.

[5] A.J. Dragt and E. Forest, "Computation of nonlinear behavior of Hamiltonian systems using Lie algebraic methods“, J. Math. Phys 24, pp. $2734-2744$ (1983).

[6] The Art and Science of Magnet Design: Selected Notes of Klaus Halbach, LBL Pub-755, Lawrence Berkeley Laboratory, Feb. 1995, p. 65.

[7] M. Venturini and A. Dragt, "Accurate Computation of Transfer Maps from Magnetic Field Data", Nucl. Inst. and Meth. A 427, pp. 387-392 (1999). 